GA-A--20641

DE92 005439

\title{
JAN 131992 \\ AN ADVANCED PLASMA CONTROL SYSTEM FOR THE DIII-D TOKAMAK
}

\author{
by \\ J.R. FERRON, A. KELLMAN, E. McKEE, T. OSBORNE, \\ P. PETRACH, T.S. TAYLOR, J. WIGHT, E. LAZARUS
}

NOVEMBER 1991

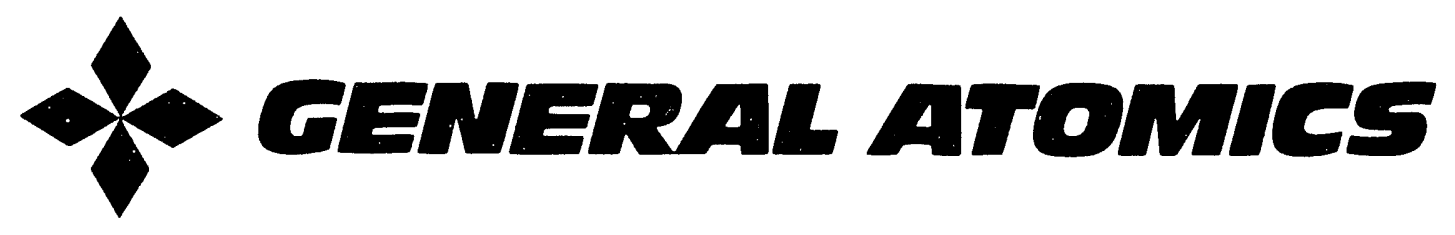




\section{DISCLAIMER}

This report was prepared as an account of work sponsored by an 'igency of the United States Government. Neither the United States Government nor any agency thereof, nor any of their employees, makes any warranty, express or implied, or assumes any legal liability or responsibility for the accuracy, completeness, or usefulness of any information, apparatus, product, or process disclosed, or represents that its use would not infringe privately owned rights. Reference herein to any specific commercial product, process, or senvice by trade name, trademark, manufacturer, or otherwise, does not necessarily consti fute or imply its endorsement, recommendation, or favoring by the United States Government or any agency thereof. The -iews and opinions of authors expressed herein do not necessarily state or reflect those of the United States Government or any agency thereof. 


\title{
AN ADVANCED PLASMA CONTROL SYSTEM FOR THE DIII-D TOKAMAK
}

\author{
by \\ J.R. FERRON, A. KELLMAN, E. McKEE, T. OSBORNE, \\ P. PETRACH, T.S. TAYLOR, J. WIGHT, E. LAZARUS*
}

*Oak Ridge National Laboratory

This is a preprint of a paper to be presented at the 14th Symposium on Fusion Engineering, September 30-October 3, 1991, San Diego, California, and to be printed in the Proceedings.

\author{
Work supported by \\ Department of Energy \\ Contract DE-AC03-89ER51114
}

GENERAL ATOMICS PROJECT 3466

NOVEMBER 1991

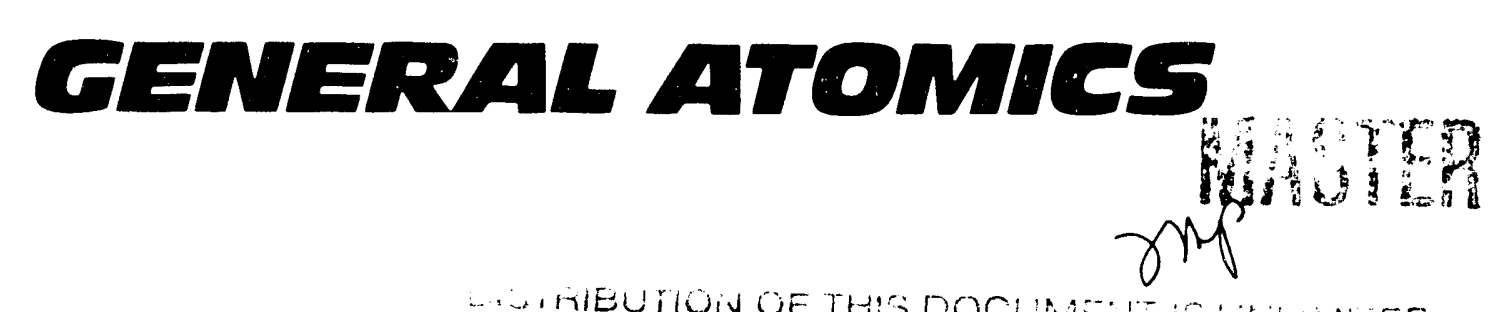




\title{
AN ADVANCED PLASMA CONTROL SYSTEM FOR THE DIII-D TOKAMAK
}

\author{
I.R. Ferron, A. Kellman, E. McKee, T. Osborne, P. Petrach, T.S. Taylor, J. Wight \\ General Atomics \\ P.0. Box 85608 \\ San Diego, California 92186-9784 \\ E. Lazarus \\ Oak Ridge National Laboratory \\ P.O. Box 2008 \\ Oak Ridge, Tennessee 37831
}

\begin{abstract}
An advanced plasms control system is being implemented for the DIII-D tokamak utilining digital technology. This system will regulate the position and shape of tolonmak discharges that range from elongated limiter to single-null divertor and double-null divertor with elongation as high as 2.6. Development of this system is expected to lead to control system technology appropriate for use on future tolarmaks such as ITER and BPX. The digital system will allow for increased precision in shape control through real time adjustment of the control algorithm to changes in the shope and discharge parameters such as $\beta_{p}, L_{i}$ and scrape-off layer current. The system will be used for research on real time optimization of discharge performance for disruption avoidance, current and preasure profile control, optimization of If antenna loading, or feedback on heat deposition patterns through divertor strike point position control, for example. Shape control with this system is baced on linearisation near a target shape of the controlled parametess a a function of the magnetic diagnootic sigmal. This digit-j oystem is unique in that it is deaigned to have the speed necesary to control the unstable vertical motion of highly elongated toknmak discharges such as those produced in DIII-D and planned for BPX and ITER. A $40 \mathrm{MBz}$ Intel i860 processor is interfaced to up to 112 channels of analog input signals. The commands to the poloidal field coils can be updated at $80 \mu$ intervals for the control of vertical position with a delay between sampling of the analog signal and update of the command of lese than $80 \mu$.
\end{abstract}

\section{Introduction}

The flexible poloidal field coil and power supply system in use on the DIII-D tokamak allows for the production of discharges with a wide variety of shapes. Elongated limiter discharges, single-null divertor discharges and double-null divertor discharges with elongation as high as 2.6 are produced routinely. Discharge shape parameters such as major radius, minor radius, elongation, triangularity, aspect ratio and X-point position are variable over a wide range. This paper describes a new plasma control system for the DIII-D tokamak that will provide improved capability to make use of this flexibility in tolcamak physics experiments.

The discharge shape is maintained by a feedback control system which uses measured magnetic field and magmetic flux values to regulate the current in the poloidal field coil set. For this control function, each discharge shape is characterized by a specific set of shape parameters. For example, five shape parameters of the single-null divertor (Fig. 1) are regulated by the present control system [1]: the radial position of the center of the separatrix flux surface $\left(\boldsymbol{R}_{\text {nurf }}\right)$, the vertical position of the current center $\left(Z_{\text {cur }}\right)$, the vertical position of the X-point $\left(Z_{v}\right)$, and the gaps between the separatrix and the inside and top wall limiters $\left(G_{A P} P_{i n}, G A P_{\text {top }}\right)$. In the present control system an analog computer which finds the difference between the actual value of a shape parameter and the intended value of the parameter is constructed from analog adders, multipliers and dividers [2] with gains and wiring configuration that are fixed during a discharge. The shape difference is used to generate commands for the poloidal field power supplies [1].

The fixed algorithm for shape computation used by the analog system must be designed to work with discharges having a wide range of values of the shape parameters. Experience has shown that on the average, with this type of algorithm a given shape parameter can be maintained close to the intended value [1]. However, there is often a noticeable error between the computed shape parameter and the actual value in the discharge because the fixed algorithm cannot match well to every discharge. In addition, variations in plasma parameters such as internal inductance $\left(\ell_{i}\right)$, stored energy (or poloidal beta $\beta_{p}$ ), or current flowing in the scrape-off-layer $\left(J_{s}\right)$ can cause changes in shape parameters which cann th be recognised correctly by a fixed control scheme, usually resulting in differencus between the actual discharge shape and the intended shape which can vary with time during a discharge as the intended shape is varied and as parameters such as $\ell_{i}, \beta_{P}$ and $J_{S}$ vary.

The shape errors can be significantly reduced by using a control scherene which is tuned for discharges with a narrow range in the shape parameters and plasms parameters. However, this requires a control algorithm which can be varied in time during

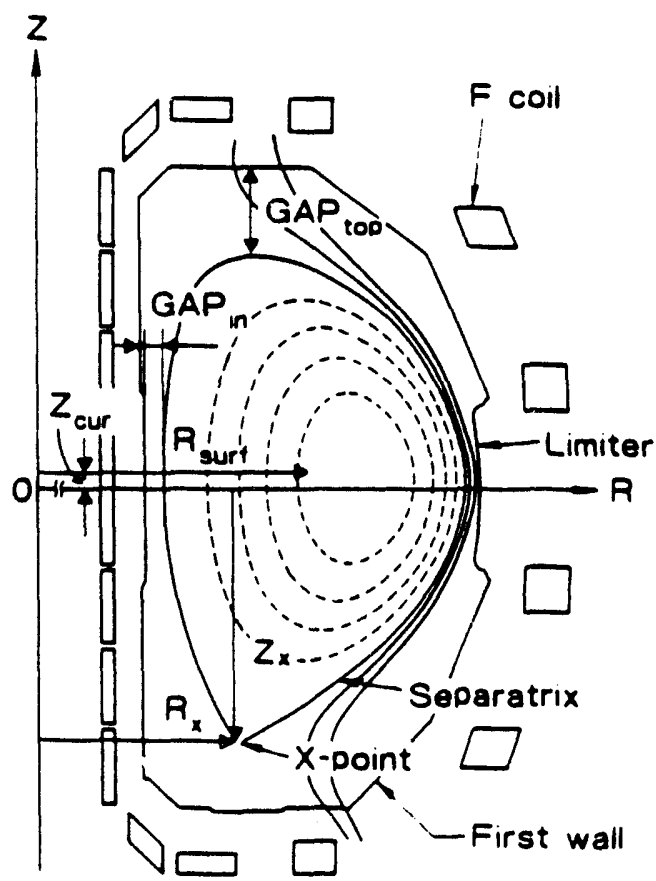

Fig. 1. Shape parameters in a single-null divertor discharge. 
a discharge as the desired shape changes and as the plasma parameters change. The present analog system is not capable of this because the gains and wiring scheme are fixed and can only be changed by hand.

This paper describes an advanced plasms control system that is being implemented for the DIII-D tolanmak which will provide the capability of variation of the control algorithm during a discharge and so will provide more precise control of the discharge shape. It will be possible to tune the control scheme in real time to the actual discharge shape and values of $\ell_{i}, \beta_{p}$, and $J_{S}$. In addition there will be improved ability to control a wider range of discharge shapes and to control shape parameters not presently regulated, increased capability for widely varying shapes in a single discharge or in successive discharges, and increased ease of operation.

In addition to control of the discharge shape, the advanced control system will be capable of optimization of discharge performance through real time application of plamma physica-based algorithms. For this functios the control system determines the required values of $10 \mathrm{me}$ discharge parameters in real time rother than strictly following a preprogrammed time evolution. One application of discharge optimization involves the specification of a parameter space to which the discharge should be restricted and action to be taken when the edge of the parameter space is approached. This is useful for avoidance of a density or beta limit or other causes of disuption. Other applications involve feedback regulation of a complex parameter such as the safety factor $(q)$, the ratio of the decay index to the critical decay index for the $n=0$ instability $\left(n / n_{e}\right)$, or the loading impedance of an if antenna. Also appropriate is control of up/down heat flux balance in a double-null divertor, control of divertor tile temperature through regulation of divertor strike point locations and control of plasma current density and presoure radial profiles through control of heating and current drive sources.

Both precision control of the discharge shape and techniques for discharge optimization are essential ingredients in a plasma control system for future tolomakes such as BPX and ITER. Development of the DIII-D advanced plasma control system described here is expected to lead to technology appropriate for use on these devices. The control system is being implemented utilizing digital technology in a way that is unique in that it is designed to have the speed necessary to control the unstable vertical motion of highly elongated tolamak discharges such as those produced in DIII-D and planned for BPX and ITER yet it still has a much simpler design than is used for digital control on other tokamaks $[3,4]$.

\section{Implementation of the Control System Hardware}

The control system, shown by block diagram in Fig. 2, has a relatively simple design: analog signals are digitized, the digital data is transmitted to a high speed microprocessor, the microprocessor executes the shape control algorithm, and the result is written to digital to analog converters which drive the controlled systems. Initially 18 poloidal field coil power supplies will be controlled but additional inputs and outputs are provided for future use in discharge optimization algorithms.

Up to 112 analog signals from tolamak diagnostics are inputs to $7 \mu \mathrm{s}$ conversion time, 12 bit $A / D$ converter modules with a separate $A / D$ converter provided for each input signal. The digitizers are CAMAC format, TRAQ system modules manufactured by DSP Technology Inc. (Table I). The TRAQ system modules have a private digitizer interface bus which is used by a custom-built digitizer controller module to control the transfer of data from the A/D converters to the real time computer. The CAMAC bus is not used except to provide power.

The real time computer is the SuperCard-2 manufactured by CSP Inc., a VME format, single board computer based on the Intel i860 RISC-design microprocessor. The processor is

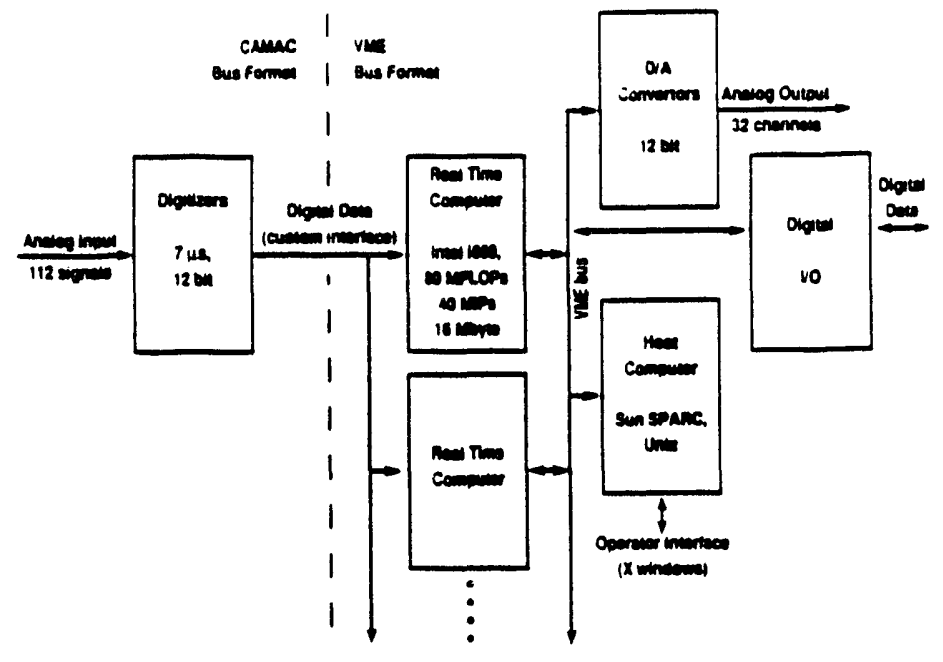

Fig. 2. Block diagram of the DIIt-D digital plasma control system.

Table I. Plasms Control System Hardware

\begin{tabular}{|c|c|}
\hline A/D converter & $\begin{array}{l}\text { DSP Technology Ine., 2812A, } 8 \text { channel/ } \\
\text { module, } 12 \text { bit, } 7 \mu \text { conversion time }\end{array}$ \\
\hline Real time proceswor & $\begin{array}{l}\text { CSP Inc. SuperCard-2, Intel i860 } \\
\text { procesies, } 16 \text { Mbyte memory. }\end{array}$ \\
\hline Daughter boerd & $\begin{array}{l}\text { General Atomies, custom design, first-in, } \\
\text { firte-out memony buffer }\end{array}$ \\
\hline D/A comverter & $\begin{array}{l}\text { DATEL Inc., DVME- } 628,8 \text { channel, } \\
12 \text { bit, } 6 \mu \text { settling time }\end{array}$ \\
\hline Hodt procenor & $\begin{array}{l}\text { Sun Mierosystems, SPARCengine IE } \\
\text { with expansion board, } 36 \text { Mbyte }\end{array}$ \\
\hline Digital 1/0 & VME Mierogysterm, VMIVME-2511 \\
\hline
\end{tabular}

capable of performing $8 \times 10^{7}$ floating point operations per second simultaneously with $4 \times 10^{7}$ scalar unit operations per second. This processing speed can be realized during vector operations and multiplication of large matrices, the operations that are required for the tolamak discharge shape control algorithm described here. This high processing speed allows the shape control function to be performed with one processor rather than multiple processors as in other schemes $[3,4]$. Provision has been made, though, for the diagnostic data to be provided simultaneously to several processors. The additional processors would be used to execute the discharge optimization algorithms.

The i860 processor utilizes a 64 bit wide data bus with a 50 ns cycle time allowing peak data transfer rates between the processor and memory of $160 \mathrm{Mbyte} / \mathrm{s}$. It is this high data transfer rate combined with the high processing speed which makes the $i 860$ a better choice for this application than specialized digital signal processors or other general purpose microprocessors. The SuperCard-2 has a daughter board port which allows an external device to transfer data directly to the main processor memory at the full $160 \mathrm{Mbyte} / \mathrm{s}$ rate. A custom daughter board has been constructed which provides the interface between the TRAQ system digitizers and the SuperCard-2. With this custom interface, data can be transferred from the digitizers to the processor memory at $4 \times 10^{7}$ samples per second so that one sample from each of the 112 data input channels can be transferred to the SuperCard memory in $2.8 \mu \mathrm{s}$. An additional $2.8 \mu \mathrm{s}$ is required to convert the data to floating point format from the integer format provided by the A/D converters. The total tim. from sampling the analog input to completion of conversion to floating point is $12.6 \mu \mathrm{s}$. This high speed data acquisition is a 
key to providing a short control cycle time with low phase shift between the input data and the output control signals.

The result of the real time calculations is transferred over the VME bus to the D/A converter modules and the analog output is used to drive the poloidal field power supplies. A digital input/output module is used for synchronization to the DIII-D discharge timing. The host computer, a Sun Microsystems SPARC architecture, VME format single board computer is used for software development and to run operator interface software. The operator interface is through X terminals which provide a graphical interface environment.

\section{The Control System Processing Cycle}

During the production of a discharge, the shape control system processor loops contimuously, executing the shape control algorithm in each cycle of the loop. A brief outline of the steps involved in a control system cycle is as follows.

1. Measurements of magnetic flux, magnetic field, dianagnetic flux, poloidal field coil current and plasma current Rogowski coil signal are made to produce the data vector $\vec{D}$. Presently $\vec{D}$ has 96 elements.

2. The data vector is used to compute values of 18 discharge shape parameters resulting in the shape vector $\vec{S} . \beta_{P}$ and $\zeta_{i}$ are also computed for a total of 20 computed values. $J_{s}$ can also be computed in this step.

3. The computed shape parameter values are compared to the target shape vector $(\vec{T})$ to generate a vector of error values: $\vec{E}=\vec{S}-\vec{T}$.

4. A total error vector is computed from terms directly proportional to $\vec{E}$, proportional to the time integral of $\vec{E}$, and proportional to the time derivative of $\vec{E}$ (a "PID" algorithm) resulting in the vector $\vec{P}$.

5. $\vec{P}$ is translated to commands to increase or decrease the current in the poloidal field coila: $\vec{I}=\vec{P} \cdot \mathbf{M}$. There are 18 poloidal field coils and 18 shape parameters so $M$ is an $18 \times 18$ matrix. M specifies which coil currents should be changed in order to adjust each of the shape parameter.

Step 2 here, computation of the discharge shape from the measured field and tlux values, is the key part of the shape control process. There are several possible algorithms for this and a digital processor, rather than an analog or analog/digital hybrid processor, was chosen specifically because of the ease with which the shape computation algorithm can be changed. Algorithma to be considered in the future include those based on neural network technology [5] or real time solution of equilibrium models.

The algorithm to be used initially is based on linearization near a specific target shape of the controlled shape parameters as a function of the magnetic field and flux values. To generate a vector of values of the shape parameters, the control system performs a multiplication of the control matrix $\mathbf{C}$ and the vector of measured data values: $\vec{S}_{1}=\vec{D}$. C. With 18 poloidal field coils in DIII-D it is in principle possitle to control 18 different shape parameters. In addition, $C$ is sed to compute $\beta_{P}$ and $\ell_{i}$. Therefore $C$ is a $96 \times 20$ matrix and $\vec{S}_{1}$ contains the 18 element shape vector $\vec{S}$ plus $\beta_{p}$ and $\ell_{i}$. The control matrix for a given target discharge shape is pre-computed using a database of computed equilibria with shapes close to the target shape. A linear least squares fit is used to find the best fit between linear combinations of the magnetic field and flux values and the shape parameters that are to be controlled.

The matrix multiplication $\vec{S}_{1}=\vec{D}$. C (Step 2 above) consumes $\sim 50 \%$ of the time used for each control system cycle. In a matrix multiplication routine the Intel $i 860$ processor can make use of instructions which allow a floating point addition, a floating point multiplication and an integer unit instruction to be completed on each clock cycle. The routine presently in use achieves 79.4 MFLOPS plus 35.5 MIPS, close to the theoretical maximum, to complete the matrix multiplication in $49.7 \mu \mathrm{s}$. The $\vec{P} \cdot$ M matrix multiplication requires $12.5 \mu \mathrm{s}$.

The remainder of the control system cycle includes the acquisition of data, described in the previous section, the PID computation, the selection of the control matrix and some other housekeeping functions. The total time for a single control system cycle as described above is $100 \mu s$ (a $10 \mathrm{kHz}$ rate).

An improvement in the control cycle is possible by exe-. cuting the control algorithm on two time scales, a fast loop for control of the unstable vertical motion and a slower loop for control of the other shape parameters which are stable and respond relatively slowly to poloidal field coil current changes. To do this, vertical position control commands can be computed several times in each computation loop for the other shape parameters. This allows the power supply commands to be updated at approximately $12.5 \mathrm{kHz}$ (80 $\mu$ intervals) for vertical position control so that frequency components up to $6.25 \mathrm{kHz}$ are retained. The power supply frequency response is $3 \mathrm{kHz}$ (in the best case which is for small signals) so the control system frequency response is larger than required. However, a short cycle time is necessary to maintain a small delay between sampling of the analog data and output of the power supply command. The delay is expected to be approximately $80 \mu \mathrm{s}$ which represents a relatively small phase shift of $14^{\circ}$ in the $500 \mathrm{~Hz}$ component. The rate at which other shape parameters can be changed is determined primarily by the skin time of the vacuum vessel which is several milliseconds. For this a $4 \mathrm{kHz}$ cycle rate is expected.

\section{The Shape Computation Algorithm}

As described in the previous section, the discharge shape is computed from a linear combination of the diagnostic signals. Figure 3 illustrates the improved accuracy of the shape calculation with this linearization technique using the vertical position of the $X$-point in a single-null divertor $\left(Z_{z}\right.$, Fig. 1) as an example. Figure 3(a) shows the actual value of $Z$, obtained from an equilibrium calculation as a function of the value computed using the non-linear algorithm implemented in the present DIII-D analog control syotem [1]. Each point on the plot represents the result for a single equilibrium. The equilibria plotted in the figure have shape parameters and plasma parameters $\left(\ell_{i}, \beta_{P}\right)$ which vary over a wide range. There is a significant amount of deviation from the ideal case illustrated by the solid line where the actual and calculated values are identical.

Figure 3(b) is a similar plot for the case where the calculated value of $Z_{\text {a }}$ is from a linear combination of the same magnetic measurements used in the calculation in Fig. 3(a), the equilibria in the database vary by a relatively small amount from the average shape, and $\beta_{P}$ and $h_{i}$ are fixed. The linear combination method applied to this restricted set of equilibria results in a standard deviation of the difference between the calculated and actual values that is a factor of 20 smaller than in Fig. 3(a).

Figure 3(c) shows the result from a linear combination of magnetic measurements as in Fig. 3(b), but the values of $\beta_{P}$ and $\ell_{i}$ vary in the range $0<\beta_{p}<1.6,0.9<\ell_{i}<1.4$. The variation in plasma parameters increases the standard deviation by a factor of 5 over the case in Fig. 3(b) illustrating the improvement that can be made by using a control matrix which is derived from equilibria having values of $\beta_{P}$ and $\ell_{i}$ in a restricted range.

The current in the scrape-off-layer is also expected to bave a strong effect on the choice of the control matrix. Figure 4 shows, for example, that the result of the equilibrium calculation of $Z_{\mathrm{z}}$ for a single-null divertor discharge is a strong function of the assumed value of the current lensity at the separatrix $\left(J_{X}\right)$. Thus, in discharges which would be expected to have significant current at the plasma edge, the calculation of the location of the plasma boundary should include a measurement of this current. 

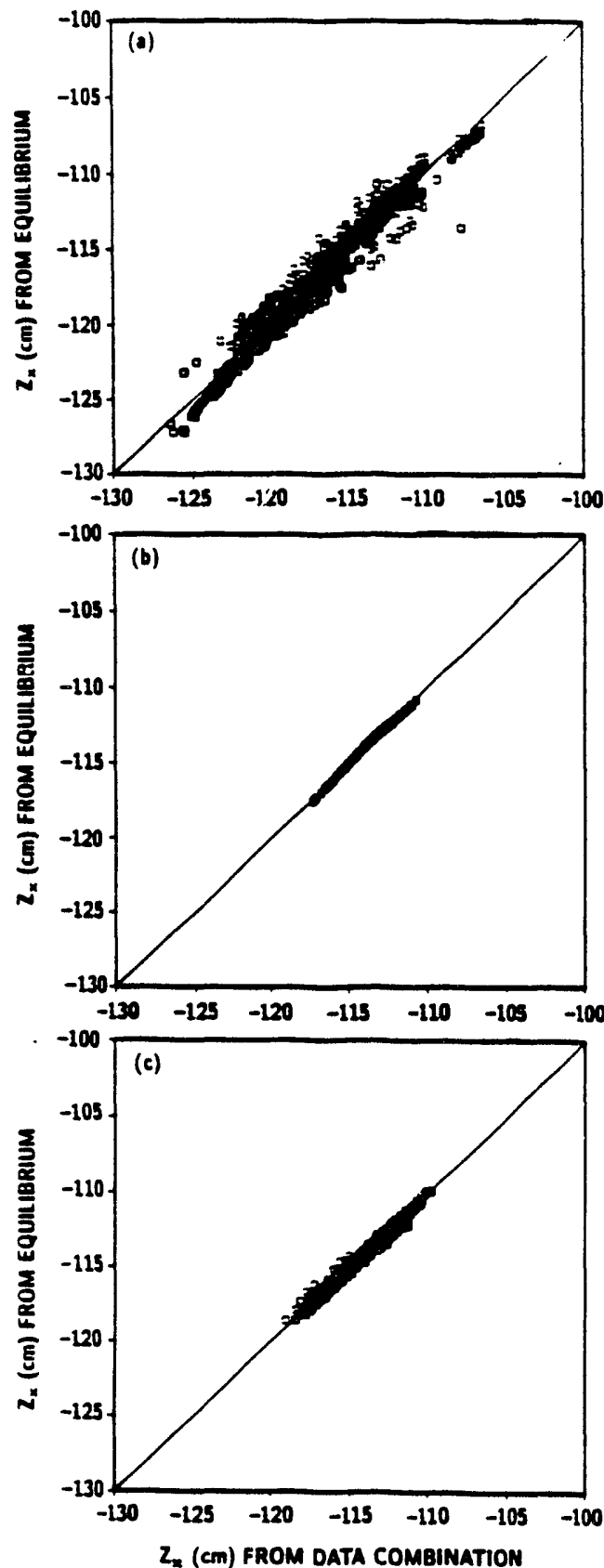

Fig. 3. Values of $Z_{2}$ (Fig. 1) obtained from a full equilibrium caleulation plotted versus values of $Z_{z}$ obtained from several shape calculation algorithms. (a) The $Z_{z}$ calculation used by the analog control system plotted for a database of discharges with widely varying singlenull shapes and values of $\beta_{P}$ and $\ell_{i}$. The standard deviation $(\sigma)$ of the difference between the equilibrium result and the control algorithm result is $0.8 \mathrm{~cm}$. (b) $Z_{2}$ calculated from a linear least squares fit with a database of discharges with constant $\boldsymbol{l}_{i}$ and $\beta_{P}$ and shapea vary-

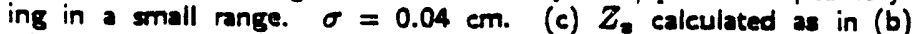
with a database which includes variation in $\ell_{i}$ and $\beta_{P} . \sigma=0.2 \mathrm{~cm}$.

A given control matrix computed by linearizing near a specified discharge shape can be used to compute the dis harge shape as long as the shape parameters and plasma parameters vary by only a small amount. In order to vary the shape par rameters within this small range the value of the target vector $\vec{T}$ is varied. In order to change the shape parameters to values outside the valid range for a given control matrix it is necessary to change the control matrix.

For each discharge shape a set of control matrices is provided, each of which is valid for a specific range of $\beta_{P}, \ell_{i}$ and

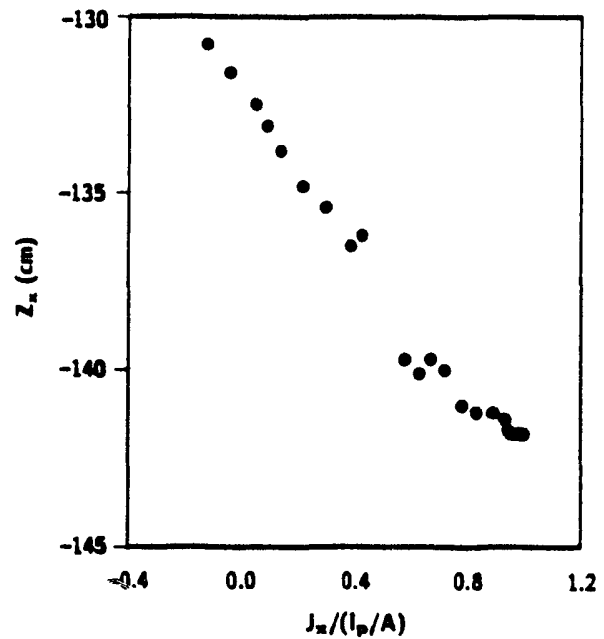

Fic. 4. The value of $Z_{2}$ (liig. 1) obtained from equilibrium calculations of a singlenull divertor discharge using a fixed set of input magnetic data as a function of the ascumed ratio of the current density at the $X$-point $\left(J_{X}\right)$ to the average current density. In this example, the best fit to the mognetic diagnostic signals is at $J_{X} /\left(I_{P} / A\right) \approx 0.46$.

$J_{S}$. At each cycle of the control system these three plasma parameters are computed and the appropriate control matrix is chosen for the next cycle. Before the production of a discharge, the real time processor is programmed with all of the control matrices necessary for control of the planned shapes and a table is provided which specifies which set of control matrices should be used for each control cycle during the discharge. In addition, the target vector can be changed at regular intervals during the discharge. Together, the preprogrammed time evolution of the control matrix set and the target vector allow the discharge shape to accurately follow a programmed time evolution.

\section{Summan}

The new, digital plasma control system for DIII-D has been described. Through use of a control algorithm which can be varied in real time this system is expected to provide more precise control of the DIII-D discharge shape than is possible in the analog control system presently in use. In addition to shape control the system will provide a platform for research on real time optimizration of discharge performance. A relatively simple design has been achieved through the use of a small number of very high speed digital processors coupled with high speed data acquisition hardware. The frequency response is expected to be adequate to control the unatable vertical motion of highly elongated discharge shapes.

\section{Acknowiedgment}

This work was sponsored by the U.S. Depertment of Energy under Contract No. DE-AC03-89ER51114.

\section{References}

[1] S. Kinoshita, H. Fukumoto, A.G. Kellman et al, General Atomics Report GA-A19584 (1990).

[2] J.S. Haskovec, A.G. Kellman, C.D. Moore, E.J. Strait, T.S. Taylor, in Proceedings of the 12th Symposium on Fusion Engineering, [Monterey, California, IEEE (1987)], p. 603.

[3] T. Kimura, K. Kurihara, M. Matsukawa et al., in Proceedings of the 12th Symposium on Fusion Engineering, (Monterey, California, IEEE (1987)], p. 565.

(4) T. Kimura, K. Kurihara, M. Takahashi et al., IEEE Transactions on Nuclear Science 36, 1554 (1989).

(5) J.B. Lister and H. Schnurrenberger, Nucl. Fusion 31, 1291 (1991). 

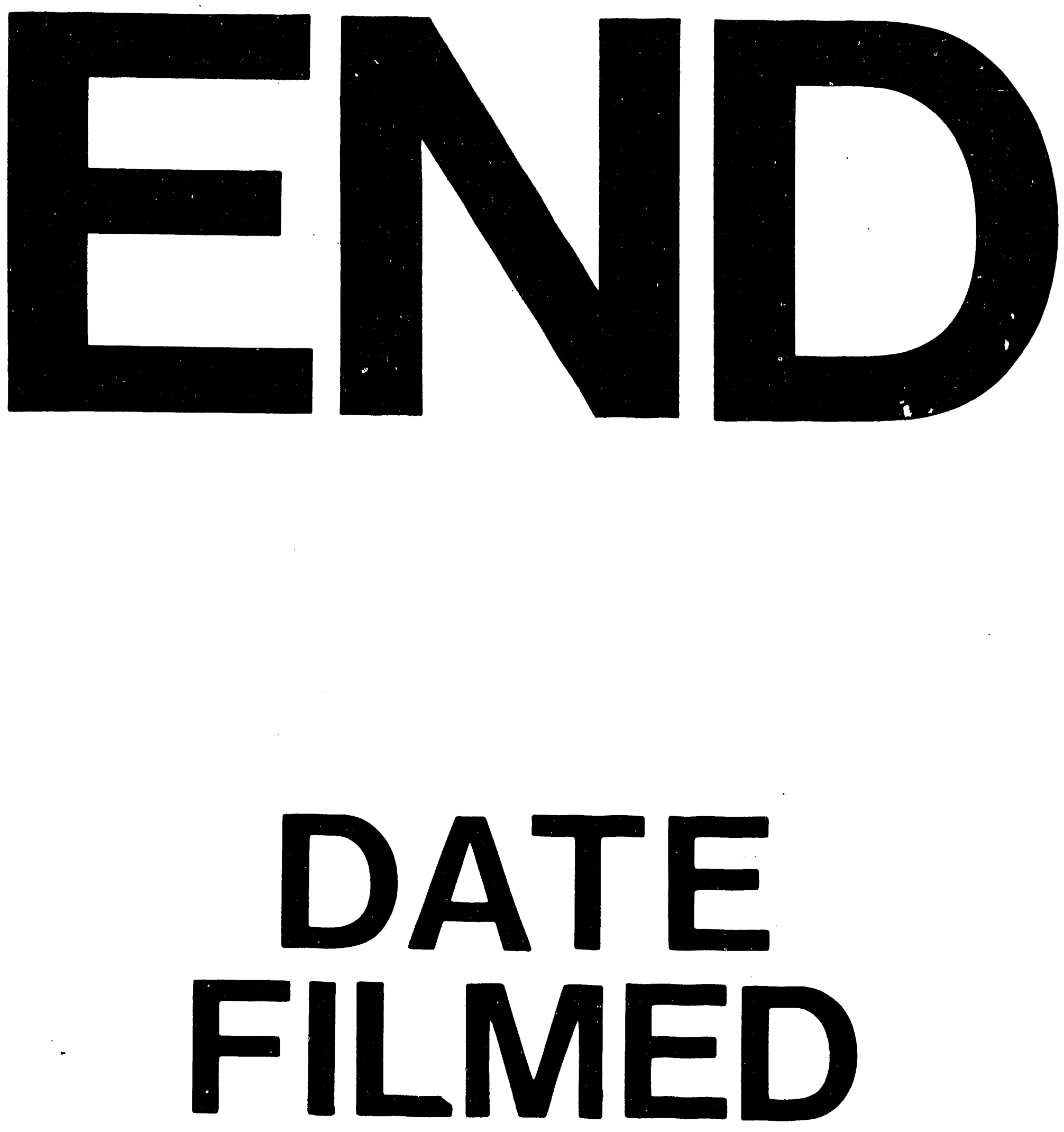

1

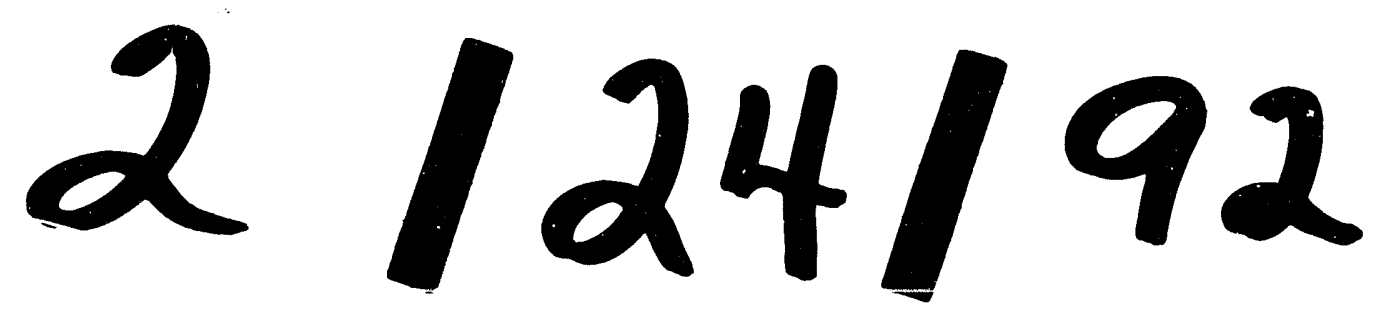


